\title{
Características Psicométricas de la Escala de Estrategias Afectivas en el Proceso de Aprendizaje (EEAA)
}

\section{Lourdes Villardón-Gallego, Concepción Yániz}

Departamento de Didáctica y Desarrollo Curricular, Universidad de Deusto, Bilbao

\section{España}

Correspondencia: Lourdes Villardón Gallego, Department of Teaching and Curriculum Development. University of Deusto, Apdo. 1, 48080 Bilbao. Spain.

(C) Education \& Psychology I+D+i and Ilustre Colegio Oficial de la Psicología de Andalucía Oriental 


\section{Resumen}

Introducción. Entre las estrategias afectivas como forma de afrontar estados afectivos vinculados al proceso de aprendizaje se incluyen aquellas orientadas al control de emociones y las enfocadas a la motivación; ambas influyen directa o indirectamente en el rendimiento. El objetivo de esta investigación fue diseñar un instrumento para medir las estrategias afectivas utilizadas por los estudiantes universitarios durante el proceso de aprendizaje coherente con el concepto de estrategia de tipo afectivo y con el modelo teórico presentado.

Método. Un total de 487 estudiantes universitarios de distintas titulaciones respondieron a la Escala de Estrategias Afectivas de Aprendizaje y a la Escala de Competencia para aprender. Para la validación del instrumento se realizaron diferentes técnicas y análisis tales como juicio de expertos, análisis factorial confirmatorio, análisis de fiabilidad y correlaciones.

Resultados. Se ha validado un instrumento de 37 item para medir las Estrategias Afectivas basado en un modelo teórico formado por cinco factores: Evitación de Esfuerzo, Imagen Social, Motivación Intrínseca, Control de Ansiedad Externo y Control de Ansiedad Interno. Todos los factores están correlacionados menos el factor Evitación de Esfuerzo que no se relaciona con Control de Ansiedad Interno y apenas se relaciona con Motivación. Es preciso seguir avanzando en el modelo y en el instrumento para mejorar el ajuste. Las correlaciones de esta escala de Estrategias Afectivas y sus dimensiones con la escala y con las dimensiones de la Competencia para Aprender avalan la validez de criterio del instrumento. Los datos confirman que la Motivación Intrínseca y el Control de Ansiedad correlacionan de forma positiva y moderada con la Autogestión del Aprendizaje, mientras que la Evitación del Esfuerzo no se relaciona con ninguna dimensión de la Competencia para Aprender.

Conclusiones. Se propone incidir en el uso de estrategias afectivas adaptativas y sustituir, en la medida de lo posible, las estrategias negativas por alternativas más eficaces para aprender.

Palabras Clave: estrategias afectivas, competencia para aprender, análisis factorial confirmatorio, enseñanza universitaria, autorregulación del aprendizaje. 


\title{
Psychometric characteristics of the EEAA (Scale of Affec- tive Strategies in the Learning Process)
}

\begin{abstract}
Introduction. Among affective strategies as a way to face affective states linked to the learning process are included those emotion-control-oriented and those motivation-control-oriented strategies. Both of them affect direct and indirectly to the learning efficiency. The objective of this research is to design an instrument for measuring the affective strategies used by the university students. This measuring model is coherent with the concept of affective strategies and with the theoretical model presented in this work.

Method. 487 students from different qualifications answered the Affective Learning Strategies Scale and the Learning Competence Scale. For the validation of this instrument different strategies were used, such as expert judgment, confirmatory factorial analysis and reliability and correlation analysis.

Results. A 37 item instrument has been validated in order to measure the Affective strategies. The measurement was based on a theoretical model consisting of 5 factors: Effort Avoidance, Social Image, Intrinsic Motivation, External Anxiety Control and Internal Anxiety Control. All these factors are correlated except the Effort Avoidance factor which is not correlated with Internal Anxiety Control and very few with Intrinsic Motivation. It is necessary to continue working on the model and the instrument to improve the statistical adjustment. The correlations of the Affective Strategies and the Learning Competence (scales and dimensions) support the criterion validity of the instrument. The results confirm that the Intrinsic Motivation and the Anxiety Control correlate in a positive and moderate way with the Learning Selfmanagement while the Effort Avoidance is not related to any dimension of the Learning Competence.
\end{abstract}

Conclusion. It is proposed the use of adaptive affective strategies, and to substitute the negative strategies by more efficient learning alternatives.

Keywords: affective strategies, learning competence, confirmatory factorial analysis, university teaching, self-regulation of learning. 


\section{Introducción}

Este artículo se enmarca en el estudio de la competencia para aprender y los diferentes factores relacionados con ella. La afectividad es un constructo psicológico que se refiere a la disposición de las personas para verse afectadas por distintos estímulos, estados o situaciones, del entorno. Páez y Carbonero (1993), al definir la afectividad, consideraban tres componentes principales, procesos motivacionales, procesos emocionales y esquemas cognitivos que se denominarán motivación, emoción y creencias. En el contexto educativo y referidos al aprendizaje, los factores afectivos se describen como las emociones, los estados de ánimo, las creencias y la motivación que influyen en cómo se perciben las situaciones de aprendizaje (Bueno, Teruel y Valero, 2005). En este mismo contexto, los enfoques de dominio afectivo sintetizan estos factores en emociones, creencias y actitudes (Gil, Blanco y Guerrero, 2005). Los elementos emocionales implicados en los procesos de aprendizaje tienen que ver con el control emocional durante el proceso de aprendizaje, desde la planificación a la evaluación; con el mantenimiento de un tono motivacional necesario para cumplimentar el proceso; con el mantenimiento del esfuerzo necesario para llevarlo a cabo, superando los estados que interfieren en tal mantenimiento; con la imagen social y con la gestión de la tarea (Zimmerman, 2011).

Los estudios sobre la dimensión afectiva del aprendizaje han crecido notablemente en los últimos años, especialmente los referidos al aprendizaje de algunas áreas como ciencias (Brígido, Caballero, Bermejo y Mellado, 2009; Garritz, 2010; Koballa y Glynn, 2007), matemáticas (Gamboa Araya, 2013; Gil, Blanco y Guerrero, 2005; 2006; Palacios, Hidalgo, Maroto y Ortega, 2013; Walshaw y Brown, 2012), lenguas (Marcos Llinàs, 2007; Marins de Andrade y Guijarro Ojeda, 2010), así como a aspectos genéricos del aprendizaje como la permanencia o abandono de los estudios (Alarcon y Edwards, 2013), y a la importancia de incluir esta temática en la formación inicial y continua del profesorado (Hugo, Sanmartí, y Adúriz Bravo 2013; Schutz y Zembylas, 2009; Zembylas, 2007).

Asumida la importancia de los procesos afectivos en el aprendizaje se considera interesante identificar actuaciones que permitan optimizar tales procesos (Boekaerts, 1995; Gargallo, Almerich, Suárez, y García-Félix, 2012). Entre dichas actuaciones se encuentran las estrategias de control afectivo que permiten regular los estados afectivos en pro de un aprendizaje exitoso. Las estrategias afectivas de aprendizaje facilitan el aprendizaje a través de la 
motivación y la emoción, a diferencia de las cognitivas que tienen una influencia directa en el proceso.

Los estudios sobre autorregulación del aprendizaje han realizado aportaciones importantes para identificar y estudiar el papel de las estrategias en la mejora de los procesos de aprendizaje. El propio concepto de autorregulación como "control que el sujeto realiza sobre sus pensamientos, acciones, emociones y motivación a través de estrategias personales para alcanzar los objetivos que ha establecido" (Panadero y Alonso-Tapia, 2014, pp. 450-451) integra las estrategias de manera nuclear. Entre dichas estrategias destacan para el objetivo de este trabajo las orientadas al control de emociones, tanto las dirigidas a disminuir la intensidad emocional que interfiere en el proceso como las enfocadas a generar emociones que soporten los procesos de aprendizaje. Estas últimas son estrategias orientadas al control de la motivación, e incluyen tanto las estrategias que suscitan el deseo de actuar antes de iniciar el proceso, como las dirigidas a mantener la concentración y el interés durante la ejecución. En adelante se denominarán estrategias afectivas a las orientadas al control de emociones y de la motivación.

La revisión de los diferentes factores afectivos influyentes en el aprendizaje permite destacar la motivación (Boza Carreño y Toscano Cruz, 2012; Gil, Bernaras, Elizalde y Arrieta, 2009; Núñez, et. al., 2009), la imagen social (Pekrun, Maier, y Elliot, 2009; Valle Arias, Nuñez Pérez et al.,2007; Valle Arias, Rodríguez Martínez et al., 2009; Vázquez, 2009; ) y la ansiedad (Álvarez, Aguilar, y Lorenzo, 2012; García-Ros, Pérez González, Pérez Blasco y Natividad, 2012; Putwain, 2007) por su impacto en el rendimiento. La relación entre metas de aprendizaje como variable motivacional asociada a la orientación intrínseca o extrínseca de la misma, las emociones y estados emocionales, particularmente la ansiedad ante el aprendizaje y los resultados de aprendizaje han generado numerosos estudios (Doménech y GómezArtiga, 2011; González, Donolo y Rinaudo, 2009; Gil, Bernaras, Elizalde, y Arrieta, 2009; Pekrun, Maier y Elliot, 2009).

Las estrategias afectivas no siempre están dirigidas al logro de objetivos deseables para el aprendizaje, adaptativos en términos psicológicos. Existen estrategias disfuncionales, ineficaces para lograr los objetivos de aprendizaje y orientadas a evitar estados desagradables (Hervás y Vázquez, 2006). En esta propuesta teórica se han incluido tanto estrategias afectivas positivas u orientadas a mejorar el aprendizaje, como negativas, orientadas a evitar esta- 
dos emocionales o situaciones afectivas desagradables, para atender a los estados afectivos (motivacionales y emocionales):

1) Motivación intrínseca: estrategias dirigidas a motivarse a uno mismo por la propia tarea.

2) Imagen social: estrategias dirigidas a cuidar la imagen social como aprendiz. Este factor está íntimamente relacionado con la orientación hacia la meta y está dirigido a preservar la propia imagen, es decir, a ser bien vistos y valorados por los demás.

3) Control de ansiedad interno (autoafirmación): estrategias dirigidas a evitar o controlar la ansiedad confiando en las propias capacidades.

4) Control de la ansiedad externo (tarea): estrategias dirigidas a pensar que se puede abordar la dificultad de la tarea utilizando procedimientos dirigidos a "minimizar" la dificultad de la tarea.

5) Evitación del Esfuerzo: estrategias dirigidas a evitar el esfuerzo. La gestión del esfuerzo refleja el compromiso de completar los objetivos del aprendizaje, a pesar de las dificultades o distracciones. Las características del aprendizaje universitario y la organización temporal del mismo hacen que esta gestión sea especialmente importante para modular el proceso y lograr los resultados pretendidos. Domenéch y Gómez-Artiga (2011) encontraron relaciones significativas y negativas entre necesidades, estrategias de evitación y rendimiento. Estos autores asignan a las estrategias de evitación un valor mediador entre las necesidades y los resultados.

El análisis del contenido de los instrumentos utilizados para medir estrategias de aprendizaje (Villardón-Gallego, Yániz, Achurra, Iraurgui y Aguilar, 2013) muestra que éstos no recogen información únicamente de estrategias, entendidas como conjunto organizado consciente e intencional de acciones o procedimientos para lograr un objetivo de aprendizaje en un determinado contexto, sino que recogen, además, estados emocionales vinculados a la situación de aprendizaje, tales como la ansiedad, o información sobre logros con relación al aprendizaje, como la capacidad para seleccionar información o para transferir lo aprendido a otras situaciones. Esta constatación ha despertado el interés por evaluar diferencialmente los estados afectivos y las estrategias utilizadas para afrontarlos. Los primeros permiten una descripción fundamentada de las emociones suscitadas durante el proceso de aprendizaje y aportan datos para interpretar posibles causas de cómo se desarrolla este proceso. Las estrategias tienen una finalidad más concreta, generar y gestionar motivos, emociones y creencias para iniciar y mantener las actuaciones necesarias para aprender. Se considera que la distinción 
entre ambos elementos permite intervenir más adecuadamente en la mejora del aprendizaje. Por este motivo se ha planteado la conveniencia de elaborar un instrumento para evaluar estrategias afectivas de aprendizaje, diferenciadas de los estados afectivos.

\section{Objetivo}

El objetivo de la investigación fue, por tanto, diseñar un instrumento para medir las estrategias afectivas utilizadas por los estudiantes universitarios durante el proceso de aprendizaje coherente con el concepto de estrategia de tipo afectivo y con el modelo teórico presentado.

\section{Método}

Participantes

La muestra está constituida por un total de 487 estudiantes universitarios de grado, de 5 facultades de la Universidad de Deusto (Bilbao, España), de los cuales 144 eran hombres y 343 mujeres. La edad media de los estudiantes era de 19.44 (D.T.=2.09), con un valor mínimo de 18 y un valor máximo de 43. Un 35.1\% cursaban primer curso y un $64.9 \%$ estaban en segundo curso de grado. Respecto a la distribución por facultades, un $31 \%$ pertenecían a la facultad de Ciencias Económicas y Empresariales, un 24.4\% a la facultad Ciencias Sociales y Humanas, un $16.6 \%$ a Derecho, un $6.8 \%$ a Ingenierías y un $21.1 \%$ a la facultad de Psicología y Educación.

\section{Procedimiento}

Se contactó con los docentes para informarles de la investigación y solicitar su colaboración para la aplicación colectiva del instrumento a los estudiantes durante una hora lectiva. Los estudiantes fueron informados de las características del estudio y del carácter voluntario de su participación. La escala fue respondida a través de una aplicación informática. Las personas que llevaron a cabo la aplicación fueron profesionales entrenadas para tal fin. El instrumento se aplicó entre los meses de Abril y Mayo de 2011.

\section{Instrumentos}

Para recoger información sobre las Estrategias Afectivas en el proceso de Aprendizaje se diseñó una escala siguiendo el modelo teórico presentado anteriormente que se ha denominado Escala de Estrategias Afectivas en el Proceso de Aprendizaje (EEAA) y cuyo proceso de elaboración y validación se presenta en este artículo. 
El instrumento fue elaborado a partir de la Escala de Evaluación de las Estrategias Motivacionales de los Estudiantes (EEMA) para universitarios, de Suárez y Fernández (2005), el cual mostró en el estudio original fiabilidades adecuadas comprendidas entre .74 y .81, y fue revisado y adaptado a estudiantes de secundaria en 2011.

Para la elaboración de la Escala de Estrategias Afectivas en el proceso de Aprendizaje (EEAA) se siguió el siguiente proceso. En primer lugar, se seleccionaron del EEMA aquellos ítems cuyo contenido se refería a una estrategia, esto es, se desecharon aquellos que se referían, por ejemplo, a un estado afectivo o a una emoción. Se seleccionaron un total de 44 ítems. En segundo lugar, se revisó y adaptó, si procedía, la redacción de los ítems seleccionados. Finalmente, se llevó a cabo un juicio de expertos para clasificar los ítems en las distintas dimensiones teóricas de las Estrategias Afectivas en el proceso de Aprendizaje: Control de Ansiedad Interno, Control de Ansiedad Externo, Evitación Esfuerzo, Motivación Intrínseca, e Imagen Social. En esta consulta participaron 7 expertos, profesores y profesoras de la Facultad de Psicología y Educación de la Universidad de Deusto, de las áreas de educación y de metodología de investigación.

Se eliminaron 3 ítems (el 14, el 22 y el 27) por discrepancia entre los jueces en su asignación a una dimensión, quedando la escala compuesta por un total de 41 ítems. Son afirmaciones en las que cada participante tiene que responder su grado de acuerdo a través de una escala tipo Likert con cinco opciones de respuesta, desde 1 (muy en desacuerdo) hasta 5 (muy de acuerdo).

Escala de Competencia para Aprender (ECA) (Villardón-Gallego, Yániz, Achurra, Iraurgui y Aguilar, 2013). La escala está compuesta por 17 ítems distribuidos en cuatro dimensiones: Transferencia del conocimiento, Conocimiento personal como aprendiz, Construcción del Conocimiento y Autogestión del Aprendizaje. Esta escala tiene una adecuada consistencia interna con una fiabilidad para la escala total de .86 y un rango de .57 a .75 para las subescalas. Asimismo, ha quedado confirmada en gran parte la estructura factorial de la misma. 


\section{Análisis Estadístico}

Para la descripción del nivel de utilización de las distintas Estrategias Afectivas durante el proceso de Aprendizaje, se calcularon medidas de tendencia central (Media - M) y de dispersión (Desviación Típica - DT). Para el análisis de los ítems del EEAA (Escala de Estrategias Afectivas de Aprendizaje) se estimó la M, DT, Asimetría (As), coeficiente de correlación del ítem con el resto de la escala (r), y valor del coeficiente alpha de Cronbach si se retirase el ítem.

Con el fin de validar el instrumento diseñado a partir del modelo teórico subyacente de la Estrategias Afectivas de Aprendizaje se llevaron a cabo diferentes procedimientos de análisis. Se puso a prueba un modelo de estructura definido a partir del marco teórico: modelo con cinco factores correlacionados. En función de los resultados de este análisis se probaron algunas variaciones de modelo con el fin de mejorar el ajuste manteniendo una propuesta teórica adecuada. Para estimar el ajuste de los modelos propuestos a los datos, se llevaron a cabo análisis factoriales confirmatorios (AFC) a partir de técnicas estructurales de covarianza, utilizando el programa EQS (Bentler, 1995; Bentler y Wu, 1995). Para la estimación de los parámetros se utilizó el método de máxima verosimilitud. En todos los casos, para evaluar el nivel de bondad de ajuste del modelo correspondiente se utilizó la prueba de ji-cuadrado $\left(\chi^{2}\right)$, que indica la probabilidad de que la divergencia entre la matriz de varianzas-covarianzas muestrales y la generada a partir del modelo hipotetizado se deban al azar. Dado que el $\chi^{2}$ es muy sensible a las variaciones del tamaño de la muestra (Schermelleh-Engel, Moosbrugger y Müller, 2003) se emplearon medidas adicionales de la bondad de ajuste del modelo (Hu y Bentler, 1999), como el error de aproximación cuadrático medio (RMSEA), el índice de bondad del ajuste (NNFI) y de ajuste comparado (CFI).

En el modelo final se indicarán los parámetros de relaciones estructurales a través de los coeficientes factoriales estandarizados y los errores de estimación. Para interpretar los resultados se eligió un nivel de significación de $p<.05$ para un intervalo de confianza del $95 \%$.

Se ha verificado la idoneidad de la matriz de correlaciones para ser factorizada a partir de la prueba de Kaiser-Meyer-Olkin y la prueba de esfericidad de Bartlett. Asimismo, se realizaron análisis de fiabilidad y de validez de criterio del instrumento. La fiabilidad fue calculada a través del alpha de Cronbach; para medir la validez de criterio de la escala se analizó la 
correlación de las puntuaciones del EEAA y de sus dimensiones con las puntuaciones de la Escala de Competencia para Aprender (Villardón-Gallego et al. 2013).

\section{Resultados}

La Tabla 1 muestra los resultados de los análisis descriptivos del conjunto de ítems de la escala EEAA. El valor de la media total es 3,31 en un rango posible de 1 a 5, siendo el valor más bajo de la media 2,54 y el máximo 3,96 para los ítems de esta escala. La asimetría de la distribución de puntuaciones en ningún ítem es mayor de $1 \mathrm{y}$, excepto en los ítems 8, 18, 26 y 34, es negativa o hacia la izquierda, lo cual indica una tendencia hacia valores más altos de la escala. La curtosis solo es mayor de 1 en el ítem 18 y en el total de la escala. Se realizó un análisis de varianza para contrastar la diferencia en la media de los ítems en función de la Facultad de pertenencia; la $\mathrm{F}$ no fue significativa en ninguno de los ítems $(p>.05)$.

Tabla 1. Estadísticos Descriptivos y Análisis de Consistencia Interna de la Escala Estrategias Afectivas en el Proceso de Aprendizaje ( $n=487)$

\begin{tabular}{|c|c|c|c|c|c|c|c|}
\hline & & $M$ & $D T$ & As & $K$ & $r$ & $\alpha$ \\
\hline 1 & $\begin{array}{l}\text { Ante las tareas me recuerdo que } \\
\text { si el profesor no es muy exígete } \\
\text { obtendré un buen resultado }\end{array}$ & 3,29 & 1,009 &,- 476 &,- 331 & ,305 & ,920 \\
\hline 2 & $\begin{array}{l}\text { Al enfrentarme con una tarea o } \\
\text { asignatura difícil me recuerdo a } \\
\text { mi mismo ocasiones similares en } \\
\text { las que finalmente no fueron tan } \\
\text { difíciles }\end{array}$ & 3,56 & ,927 &,- 590 & , 174 & ,375 & ,919 \\
\hline 3 & $\begin{array}{l}\text { Cuando no me apetece mucho } \\
\text { trabajar, comienzo con aquellas } \\
\text { partes del trabajo que me resultan } \\
\text { más agradables. }\end{array}$ & 3,96 & ,926 &,- 947 & 900 & , 169 & ,921 \\
\hline 4 & $\begin{array}{l}\text { Ante una tarea, me planteo que } \\
\text { mi objetivo será aprender cosas } \\
\text { nuevas }\end{array}$ & 3,41 & ,952 &,- 409 &,- 180 & ,477 & ,918 \\
\hline 5 & $\begin{array}{l}\text { Antes de iniciar una tarea com- } \\
\text { plicada, suelo pensar en lo intere- } \\
\text { sante que ésta puede ser }\end{array}$ & 3,17 & 1,070 &,- 228 &,- 572 & ,466 & ,918 \\
\hline 6 & $\begin{array}{l}\text { Me propongo hacer las tareas } \\
\text { mejor que los demás }\end{array}$ & 3,28 & 1,089 &,- 375 &,- 546 & ,475 & ,918 \\
\hline 7 & $\begin{array}{l}\text { Me propongo como objetivo el } \\
\text { no quedar en ridículo o parecer } \\
\text { poco capaz ante los demás }\end{array}$ & 3,22 & 1,160 &,- 337 &,- 781 & ,422 & ,918 \\
\hline 8 & $\begin{array}{l}\text { Intento evitar las tareas o asigna- } \\
\text { turas difíciles }\end{array}$ & 2,66 & 1,130 & , 188 &,- 875 & ,247 & ,921 \\
\hline 9 & $\begin{array}{l}\text { Suelo buscar que mis amigos o } \\
\text { compañeros realicen valoraciones } \\
\text { positivas sobre mi actividad } \\
\text { académica }\end{array}$ & 3,25 & 1,058 &,- 442 &,- 509 & ,502 & ,918 \\
\hline 10 & En el transcurso de las activida- & 3,13 & 1,047 &,- 304 &,- 609 &, 572 & ,917 \\
\hline
\end{tabular}




\begin{tabular}{|c|c|c|c|c|c|c|c|}
\hline & $\begin{array}{l}\text { des académicas, suelo parar y me } \\
\text { digo a mi mismo que lo estoy } \\
\text { haciendo bien y alabo mi trabajo }\end{array}$ & & & & & & \\
\hline 11 & $\begin{array}{l}\text { Cuando me encuentro con difi- } \\
\text { cultades en una tarea me digo que } \\
\text { no es necesario preocuparse, que } \\
\text { más tarde seguramente veré las } \\
\text { cosas más claras }\end{array}$ & 3,43 & ,947 &,- 523 &,- 136 & ,496 & ,918 \\
\hline 12 & $\begin{array}{l}\text { Ante las tareas, me planteo que si } \\
\text { éstas no son difíciles obtendré un } \\
\text { buen resultado }\end{array}$ & 3,37 & ,968 &,- 636 &,- 018 & ,449 & ,918 \\
\hline 13 & $\begin{array}{l}\text { Al enfrentarme con una tarea o } \\
\text { asignatura me recuerdo a mi } \\
\text { mismo que soy capaz de esfor- } \\
\text { zarme lo suficiente para tener } \\
\text { éxito }\end{array}$ & 3,73 &, 891 &,- 725 & ,618 & ,488 & ,918 \\
\hline 14 & Eliminado & & & & & & \\
\hline 15 & $\begin{array}{l}\text { Ante una tarea me planteo que } \\
\text { hacerla me resultará gratificante }\end{array}$ & 3,41 & ,977 &,- 470 &,- 090 &, 514 & ,917 \\
\hline 16 & $\begin{array}{l}\text { Me propongo como objetivo el } \\
\text { contestar a preguntas o hacer } \\
\text { tareas que los demás no saben }\end{array}$ & 3,07 & 1,061 &,- 169 &,- 599 &, 450 & ,918 \\
\hline 17 & $\begin{array}{l}\text { Cuando participo en clase intento } \\
\text { no parecer poco capaz a mis } \\
\text { compañeros. }\end{array}$ & 3,32 & 1,061 &,- 396 &,- 483 & ,262 &, 921 \\
\hline 18 & $\begin{array}{l}\text { Me propongo como objetivo el } \\
\text { aprobar, pero intentando trabajar } \\
\text { lo menos posible }\end{array}$ & 2,58 & 1,251 & ,249 & $-1,06$ &, 507 & ,917 \\
\hline 19 & $\begin{array}{l}\text { Para sentirme satisfecho con mi } \\
\text { aprendizaje busco que otros reco- } \\
\text { nozcan mis esfuerzos }\end{array}$ & 3,20 & 1,070 &,- 367 &,- 552 & ,452 & ,918 \\
\hline 20 & $\begin{array}{l}\text { Ante una tarea compleja me digo } \\
\text { a mi mismo que soy capaz de } \\
\text { esforzarme lo suficiente como } \\
\text { para hacerla bien }\end{array}$ & 3,70 & ,898 &,- 782 & ,725 & ,378 & ,919 \\
\hline 21 & $\begin{array}{l}\text { Cuando me encuentro en dificul- } \\
\text { tades trato de no preocuparme } \\
\text { por si cometo errores }\end{array}$ & 3,26 & ,966 &,- 303 &,- 389 &, 527 & ,917 \\
\hline 22 & Eliminado & & & & & & \\
\hline 23 & $\begin{array}{l}\mathrm{Al} \text { enfrentarme con una tarea o } \\
\text { asignatura difícil me recuerdo a } \\
\text { mi mismo que tengo la capacidad } \\
\text { necesaria para tener éxito }\end{array}$ & 3,64 & ,907 &,- 702 & ,479 &, 421 & ,918 \\
\hline 24 & $\begin{array}{l}\text { Me propongo conseguir califica- } \\
\text { ciones más altas que las de mis } \\
\text { compañeros }\end{array}$ & 3,08 & 1,163 &,- 101 &,- 863 &, 340 & ,919 \\
\hline 25 & $\begin{array}{l}\text { Cuando respondo a preguntas del } \\
\text { profesor intento evitar quedar mal } \\
\text { ante mis compañeros }\end{array}$ & 3,43 & 1,057 &,- 594 &,- 273 &, 517 & ,917 \\
\hline 26 & $\begin{array}{l}\text { Selecciono aquellas asignaturas o } \\
\text { tareas con las que pueda aprobar } \\
\text { trabajando lo menos posible }\end{array}$ & 2,69 & 1,138 &, 184 &,- 919 & ,447 & ,918 \\
\hline 27 & Eliminado & & & & & & \\
\hline 28 & $\begin{array}{l}\text { Para sentirme satisfecho con mi } \\
\text { aprendizaje, busco ser elogiado } \\
\text { por mis padres y/o profesores }\end{array}$ & 3,16 & 1,109 &,- 324 &,- 699 &, 508 & ,918 \\
\hline
\end{tabular}




\begin{tabular}{|c|c|c|c|c|c|c|c|}
\hline 29 & $\begin{array}{l}\text { Me motivo a mí mismo dándome } \\
\text { ánimos }\end{array}$ & 3,58 & ,982 &,- 720 & ,273 & ,547 & 917 \\
\hline 30 & $\begin{array}{l}\text { Cuando me encuentro con difi- } \\
\text { cultades durante el aprendizaje } \\
\text { trato de no preocuparme por el } \\
\text { resultado y de tener pensamientos } \\
\text { positivos }\end{array}$ & 3,41 & ,967 &,- 534 &,- 097 & ,518 & ,917 \\
\hline 31 & $\begin{array}{l}\text { Al enfrentarme con una tarea } \\
\text { difícil me recuerdo a mis mismo } \\
\text { ocasiones similares en las que } \\
\text { tuve éxito }\end{array}$ & 3,61 & ,922 &,- 740 & ,388 & ,356 & 919 \\
\hline 32 & $\begin{array}{l}\text { Antes de iniciar una tarea pienso } \\
\text { que mi objetivo es el de aprender } \\
\text { a resolver los problemas que me } \\
\text { proponen }\end{array}$ & 3,43 & ,911 &,- 335 &,- 273 & ,488 & ,918 \\
\hline 33 & $\begin{array}{l}\text { Procuro que mis compañeros no } \\
\text { se den cuenta de mis errores }\end{array}$ & 2,98 & 1,088 &,- 156 &,- 745 & ,589 & ,917 \\
\hline 34 & $\begin{array}{l}\text { Me propongo como objetivo } \\
\text { trabajar lo menos posible en clase } \\
\text { o en casa }\end{array}$ & 2,54 & 1,187 & ,339 &,- 912 & ,540 & ,917 \\
\hline 35 & $\begin{array}{l}\text { Antes de iniciar las tareas suelo } \\
\text { resaltar aquellos aspectos que me } \\
\text { resultan más novedosos }\end{array}$ & 3,31 & 999 &,- 346 &,- 286 & ,418 & ,918 \\
\hline 36 & $\begin{array}{l}\text { Intento que otros reconozcan mi } \\
\text { capacidad para así sentirme más } \\
\text { satisfecho }\end{array}$ & 3,23 & 1,022 &,- 259 &,- 607 &, 520 & ,917 \\
\hline 37 & $\begin{array}{l}\text { Ante una tarea complicada me } \\
\text { digo que tengo la capacidad sufi- } \\
\text { ciente para hacerla }\end{array}$ & 3,64 & 914 &,- 754 &, 591 & ,578 & ,917 \\
\hline 38 & $\begin{array}{l}\text { Para evitar ponerme nervioso } \\
\text { cuando realizo tareas académicas } \\
\text { trato de centrarme y de no pre- } \\
\text { ocuparme por el resultado }\end{array}$ & 3,40 & ,950 &,- 460 &,- 296 & ,550 & ,917 \\
\hline 39 & $\begin{array}{l}\text { Antes de iniciar una tarea com- } \\
\text { pleja me pongo como objetivo } \\
\text { mejorara mis habilida- } \\
\text { des/capacidades }\end{array}$ & 3,44 & ,940 &,- 461 &,- 070 & ,322 & ,919 \\
\hline 40 & $\begin{array}{l}\text { Me pongo como objetivo el no } \\
\text { quedar mal académicamente ante } \\
\text { mi familia o los profesores }\end{array}$ & 3,13 & 1,130 &,- 136 &,- 854 & ,454 & ,918 \\
\hline 41 & $\begin{array}{l}\text { Ante una tarea difícil me digo } \\
\text { que obtendré resultados positivos }\end{array}$ & 3,49 & ,979 &,- 544 &,- 049 & ,518 & ,917 \\
\hline 42 & $\begin{array}{l}\text { Cuando me aburro en las tareas } \\
\text { académicas me obligo a mí mis- } \\
\text { mo a prestar mayor atención }\end{array}$ & 3,45 & 963 &,- 502 &,- 253 & ,322 & ,919 \\
\hline 43 & $\begin{array}{l}\text { Ante las tareas difíciles me digo a } \\
\text { mi mismo que si me esfuerzo } \\
\text { obtendré resultados positivos }\end{array}$ & 3,70 & 891 &,- 862 & ,924 &, 454 & ,918 \\
\hline \multirow[t]{2}{*}{44} & $\begin{array}{l}\text { Antes de iniciar las tareas acadé- } \\
\text { micas suelo buscar los aspectos } \\
\text { más interesantes o que me plan- } \\
\text { tean un desafío }\end{array}$ & 3,34 & 947 &,- 329 &,- 280 & ,518 & ,917 \\
\hline & Escala & 3,31 & 0,50 & -.282 & 1,063 & & \\
\hline
\end{tabular}


Con relación a la consistencia interna de la escala, los coeficientes de correlación entre los ítems y la escala total están entre .17 y .59, siendo el valor promedio de correlación .45. La eliminación de los ítems 3, 8 y 18 mejoraría solo un 0,001 la fiabilidad del total de la escala, por lo que se decidió mantenerlos en este momento del análisis.

Tanto la prueba KMO (= .913) como el test de esfericidad de Bartlett $\left(\chi^{2}=8835.1 ; p<\right.$ 0.001) llevados a cabo sobre la matriz de correlaciones, indicaron que la factorización de los ítems es adecuada para la EEAA. Las pruebas de Parallel calculadas sobre la matriz de correlaciones indicaron que deberían retenerse cinco factores.

Para validar el constructo dimensional de la EEAA, se probó un modelo estructural consistente en cinco factores correlacionados (M1a): Evitación de Esfuerzo, Imagen Social, Motivación Intrínseca, Control de Ansiedad Interno y Control de Ansiedad Externo. Se llevó a cabo un análisis factorial confirmatorio para probar la estructura del modelo teórico, cuyos índices de bondad de ajuste se muestran en la Tabla 2. En función de los resultados obtenidos se modificó el modelo para mejorarlo.

Debido a que no existe normalidad multivariada en los datos (el estimador estandarizado de la curtosis multivariada de Mardia es igual a $75.76>1.96$ ), se utilizaron estimadores robustos de máxima verosimilitud. La Ji cuadrado de Satorra-Bentler $\left(\chi^{2}\right.$, como medida de ajuste general) ha sido estadísticamente significativa, lo que indica que el modelo empírico no tiene buen ajuste con el modelo teórico. Sin embargo, el valor $\chi^{2}$ tiende a aumentar en muestras amplias $(n>100)$, debido al error en la especificación del modelo (Jöreskog y Sörbom, 1989). Por ello, conviene calcular índices adicionales.

Tabla 2. Análisis Factorial Confirmatorio $(\mathrm{n}=487)$ del Modelo teórico de la Escala de Estrategias Afectivas de Aprendizaje (EEAA). Estimadores Robustos de Máxima Verosimilitud.

\begin{tabular}{|c|c|c|c|c|c|c|c|c|c|}
\hline \multirow{2}{*}{\multicolumn{2}{|c|}{ Modelo (M) de $L C S$}} & \multicolumn{8}{|c|}{ Índices de bondad de ajuste } \\
\hline & & \multirow{2}{*}{$\frac{\chi^{2}}{1726.4}$} & \multirow{2}{*}{$\frac{\chi^{2} / g l}{2.24}$} & \multirow{2}{*}{$\frac{A I C}{188.488}$} & \multirow{2}{*}{$\frac{N N F I}{.83}$} & \multirow{2}{*}{$\frac{C F I}{.84}$} & \multirow{2}{*}{$\begin{array}{c}S R M R \\
.069\end{array}$} & \multicolumn{2}{|c|}{ RMSEA $90 \% \mathrm{CI}$} \\
\hline M1a & $\begin{array}{l}\text { Cinco factores } \\
\text { correlacionados }\end{array}$ & & & & & & & {$[.047$} & $.054]$ \\
\hline M1b & $\begin{array}{l}\text { Cinco factores corre- } \\
\text { lacionados sin ítems } \\
1,3,21,42\end{array}$ & 1422 & 2,30 & 184.045 & .85 & .86 & .067 & {$[.048$} & $.055]$ \\
\hline M1c & $\begin{array}{l}\text { M1b (excepto corre- } \\
\text { laciones F1 con F3 y } \\
\text { F1 con F4) }\end{array}$ & 1428.9 & 2.30 & 186.943 & .85 & .86 & .068 & {$[.050$} & $.055]$ \\
\hline
\end{tabular}


$\chi^{2}-\mathrm{Ji}$ cuadrado

$\chi^{2} / \mathrm{gl}$ - Ji cuadrado normado: ji entre grados lib

$A I C$ - Criterio de información de Akaike

$N N F I$-Indice de ajuste No Normado

$C F I$-Índice de ajuste comparativo

$S R M R$ - Raíz cuadrada de la media de residuos estandarizados

RMSEA - Error de aproximación cuadrático medio

$90 \%$ CI -Intervalo de confianza

Fijándose en el valor de $\chi^{2}$ normado $\left(\chi^{2} / \mathrm{gl}\right)$ en la Tabla 2 , se puede observar que en el modelo analizado es mayor que 1 y menor de 3, esto es, está entre valores aceptables. Los valores de los índices NNFI y CFI son cercanos al criterio mínimo de un buen ajuste (.90). Los valores de SMRM y RMSEA, son aceptables <.08).

Con el fin de mejorar el modelo, se decidió eliminar los ítems que tenían una raíz cuadrada de R menor de .20 en la solución estandarizada (M1b). Estos ítems eran el 1 (.12), el 2 (.19), el 3 (.023), el 21 (.16), y el 42 (.15). Se decidió mantener el ítem 2 debido a que la raíz cuadrada de $\mathrm{R}$ estaba cerca de .20 y a que el ítem pertenece a una dimensión compuesta por un escaso número de ítems. Como puede observarse en la Tabla 2, los índices NNFI y CFI mejoran aunque sin alcanzar la puntuación de 90 . El índice AIC y la comparación a través de $\chi^{2}(150)=304,45(p<.01)$ indican la mejora del modelo en términos de parsimonia.

El siguiente paso para mejorar el modelo fue eliminar las correlaciones entre factores menores de .30. Así se planteó una variación del modelo M1b con todos los factores correlacionados pero eliminando la correlación de F1 con F5 $(r=.090, p<.05)$ y de F1 con F4 $(r=-$ $.016, p<.05)$. Ni los índices NNFI y CFI, ni la Ji cuadrado normada ni RMSEA varían con respecto al M1b. El resto de los índices experimenta una variación menor. La prueba de la diferencia escalada de Ji cuadrado entre los dos modelos entre M1c y M1b no es estadísticamente significativa al 99\%. Siendo, por tanto, similares en cuanto a ajuste el M1b y M1c se decide retener el M1c por coherencia con el modelo teórico, al considerar el contenido del F1 (Evitación del Esfuerzo) y del F5 (Control Ansiedad Interno) y F4 (Motivación). Los pesos factoriales y los errores de estimación se presentan en la Tabla 3. Se puede observar que los pesos factoriales (coeficientes lambda) son siempre mayores de .45 , excepto en el ítem 2 ( $\lambda=$ $.43)$.

Tabla 3. Pesos factoriales de los ítems del EEAA $(n=487)$ 


\begin{tabular}{|c|c|c|c|}
\hline Dimension & Item & \multicolumn{2}{|c|}{ Lambda-Y Error } \\
\hline \multirow{4}{*}{$\begin{array}{l}\text { Evitación de } \\
\text { Esfuerzo }\end{array}$} & 8. Intento evitar las tareas o asignaturas difíciles & 0.70 & 0.71 \\
\hline & 18. Me propongo como objetivo el aprobar, pero intentando trabajar lo menos posible & 0.78 & 0.63 \\
\hline & 26. Selecciono aquellas asignaturas o tareas con las que pueda aprobar trabajando lo menos posible & 0.81 & 0.58 \\
\hline & 34. Me propongo como objetivo trabajar lo menos posible en clase o en casa & 0.82 & 0.57 \\
\hline \multirow{12}{*}{ Imagen social } & 6. Me propongo hacer las tareas mejor que los demás & 0.55 & 0.84 \\
\hline & 7. Me propongo como objetivo el no quedar en ridículo o parecer poco capaz ante los demás & 0.60 & 0.80 \\
\hline & $\begin{array}{l}\text { 9. Suelo buscar que mis amigos o compañeros realicen valoraciones positivas sobre mi actividad } \\
\text { académica }\end{array}$ & 0.64 & 0.77 \\
\hline & 16. Me propongo como objetivo el contestar a preguntas o hacer tareas que los demás no saben & 0.52 & 0.85 \\
\hline & 17. Cuando participo en clase intento no parecer poco capaz a mis compañeros. & 0.64 & 0.77 \\
\hline & 19. Para sentirme satisfecho con mi aprendizaje busco que otros reconozcan mis esfuerzos & 0.68 & 0.74 \\
\hline & 24. Me propongo conseguir calificaciones más altas que las de mis compañeros & 0.61 & 0.80 \\
\hline & 25. Cuando respondo a preguntas del profesor intento evitar quedar mal ante mis compañeros & 0.64 & 0.77 \\
\hline & 28. Para sentirme satisfecho con mi aprendizaje, busco ser elogiado por mis padres y/o profesores & 0.65 & 0.76 \\
\hline & 33. Procuro que mis compañeros no se den cuenta de mis errores & 0.66 & 0.76 \\
\hline & 36. Intento que otros reconozcan mi capacidad para así sentirme más satisfecho & 0.74 & 0.68 \\
\hline & 40. Me pongo como objetivo el no quedar mal académicamente ante mi familia o los profesores & 0.73 & 0.68 \\
\hline \multirow{3}{*}{$\begin{array}{l}\text { Control de ansie- } \\
\text { dad externo (tarea) }\end{array}$} & $\begin{array}{l}\text { 2. Al enfrentarme con una tarea o asignatura difícil me recuerdo a mí mismo ocasiones similares en } \\
\text { las que finalmente no fueron tan difíciles }\end{array}$ & 0.43 & 0.90 \\
\hline & $\begin{array}{l}\text { 11. Cuando me encuentro con dificultades en una tarea me digo que no es necesario preocuparse, que } \\
\text { más tarde seguramente veré las cosas más claras }\end{array}$ & 0.69 & 0.72 \\
\hline & 12. Ante las tareas, me planteo que si éstas no son difíciles obtendré un buen resultado & 0.49 & 0.87 \\
\hline \multirow{7}{*}{$\begin{array}{l}\text { Motivación intrín- } \\
\text { seca }\end{array}$} & 4. Ante una tarea, me planteo que mi objetivo será aprender cosas nuevas & 0.62 & 0.78 \\
\hline & 5. Antes de iniciar una tarea complicada, suelo pensar en lo interesante que ésta puede ser & 0.62 & 0.78 \\
\hline & 15. Ante una tarea me planteo que hacerla me resultará gratificante & 0.58 & 0.81 \\
\hline & $\begin{array}{l}\text { 32. Antes de iniciar una tarea pienso que mi objetivo es el de aprender a resolver los problemas que } \\
\text { me proponen }\end{array}$ & 0.71 & 0.70 \\
\hline & 35. Antes de iniciar las tareas suelo resaltar aquellos aspectos que me resultan más novedosos & 0.57 & 0.82 \\
\hline & $\begin{array}{l}\text { 39. Antes de iniciar una tarea compleja me pongo como objetivo mejorara mis habilida- } \\
\text { des/capacidades }\end{array}$ & 0.71 & 0.71 \\
\hline & $\begin{array}{l}\text { 44. Antes de iniciar las tareas académicas suelo buscar los aspectos más interesantes o que me plan- } \\
\text { tean un desafío }\end{array}$ & 0.65 & 0.76 \\
\hline \multirow{11}{*}{$\begin{array}{l}\text { Control ansiedad } \\
\text { interno (por auto- } \\
\text { afirmación) }\end{array}$} & $\begin{array}{l}\text { 10. En el transcurso de las actividades académicas, suelo parar y me digo a mi mismo que lo estoy } \\
\text { haciendo bien y alabo mi trabajo }\end{array}$ & 0.57 & 0.82 \\
\hline & $\begin{array}{l}\text { 13. Al enfrentarme con una tarea o asignatura me recuerdo a mí mismo que soy capaz de esforzarme } \\
\text { lo suficiente para tener éxito }\end{array}$ & 0.69 & 0.73 \\
\hline & $\begin{array}{l}\text { 20. Ante una tarea compleja me digo a mi mismo que soy capaz de esforzarme lo suficiente como para } \\
\text { hacerla bien }\end{array}$ & 0.68 & 0.73 \\
\hline & $\begin{array}{l}\text { 23. Al enfrentarme con una tarea o asignatura difícil me recuerdo a mí mismo que tengo la capacidad } \\
\text { necesaria para tener éxito }\end{array}$ & 0.72 & 0.69 \\
\hline & 29. Me motivo a mí mismo dándome ánimos & 0.63 & 0.77 \\
\hline & $\begin{array}{l}\text { 30. Cuando me encuentro con dificultades durante el aprendizaje trato de no preocuparme por el } \\
\text { resultado y de tener pensamientos positivos }\end{array}$ & 0.57 & 0.82 \\
\hline & $\begin{array}{l}\text { 31. Al enfrentarme con una tarea difícil me recuerdo a mí mismo ocasiones similares en las que tuve } \\
\text { éxito }\end{array}$ & 0.67 & 0.74 \\
\hline & 37. Ante una tarea complicada me digo que tengo la capacidad suficiente para hacerla & 0.75 & 0.66 \\
\hline & $\begin{array}{l}\text { 38. Para evitar ponerme nervioso cuando realizo tareas académicas trato de centrarme y de no preocu- } \\
\text { parme por el resultado }\end{array}$ & 0.48 & 0.87 \\
\hline & 41. Ante una tarea difícil me digo que obtendré resultados positivos & 0.66 & 0.75 \\
\hline & 43. Ante las tareas difíciles me digo a mi mismo que si me esfuerzo obtendré resultados positivos & 0.66 & 0.76 \\
\hline
\end{tabular}

Los coeficientes de correlación entre los factores se recogen en la Tabla 4; todos ellos, excepto el coeficiente de correlación entre el Factor 1 Evitación y el Factor 5 Control de An- 
siedad Interno $(p>.05)$, son significativos $(p<.01)$. El Factor 4 Motivación Intrínseca tiene una correlación alta positiva con el F5 Control de Ansiedad Interno y con el F3 Control de Ansiedad Externo. El Factor 3 Control de Ansiedad Externo tiene una correlación positiva moderada con el F5 Control de Ansiedad Interno. El Factor 1 Evitación tiene una correlación positiva y moderada con el F2 Imagen Social y positiva y baja con el F3 Control Ansiedad Externa. Por su parte, el F2 Imagen Social tiene una relación positiva y baja con el F4 Motivación Intrínseca, y una relación positiva y moderada con el F3 Control Ansiedad Externo y con el F5 Control Ansiedad Interno.

Tabla 4. Coeficientes de correlación entre Factores del EEAA

\begin{tabular}{cccccc}
\hline & $\begin{array}{c}\text { F1 Evitacion } \\
\text { Esfuerzo }\end{array}$ & $\begin{array}{c}\text { F2 Imagen } \\
\text { Social }\end{array}$ & $\begin{array}{c}\text { F3 Control an- } \\
\text { siedad externo }\end{array}$ & $\begin{array}{c}\text { F4 Motivación } \\
\text { intrínseca }\end{array}$ & $\begin{array}{c}\text { F5 Control an- } \\
\text { siedad interno }\end{array}$ \\
\hline $\begin{array}{c}\text { F1 Evitación } \\
\text { Esfuerzo }\end{array}$ & --- & $.423^{* *}$ & $.331^{* *}$ & $.149^{* *}$ & .080 \\
\hline $\begin{array}{c}\text { F2 Imagen } \\
\text { Social }\end{array}$ & -- & --- & $.437^{* *}$ & $.395^{* *}$ & $.431^{* *}$ \\
\hline $\begin{array}{c}\text { F3 Control } \\
\text { ansiedad exter- } \\
\text { no }\end{array}$ & -- & -- & --- & $.802^{* *}$ & $.652^{* *}$ \\
\hline $\begin{array}{c}\text { F4 Motivación } \\
\text { intrínseca }\end{array}$ & -- & -- & -- & $.809^{* *}$ \\
\hline $\begin{array}{c}\text { F5 Control } \\
\text { ansiedad inter- } \\
\text { no }\end{array}$ & -- & -- & -- & - \\
\hline$* * p<.01$ & & & & \\
\hline
\end{tabular}

El coeficiente alpha de la escala total es .92, y los de cada dimensión son los siguientes: Evitación de Esfuerzo $(\alpha=.82)$, Imagen Social $(\alpha=.89)$, Control Ansiedad Externo $(\alpha=.54)$, Motivación Intrínseca $(\alpha=.82)$, Control Ansiedad Interno $(\alpha=.88)$. Todos ellos son adecuados; el menor es el de Control de Ansiedad Externo, aunque este resultado se puede explicar por el hecho de que esta dimensión está compuesta solo por tres ítems. 
Tabla 5. Coeficientes de correlación entre Factores del EEAA y del ECA

\begin{tabular}{cccccc}
\hline ECA/EEAA & $\begin{array}{c}\text { F1 Evitacion } \\
\text { Esfuerzo }\end{array}$ & $\begin{array}{c}\text { F2 Imagen } \\
\text { Social }\end{array}$ & $\begin{array}{c}\text { F3 Control } \\
\text { ansiedad exter- } \\
\text { no }\end{array}$ & $\begin{array}{c}\text { F4 Motivación } \\
\text { intrínseca }\end{array}$ & $\begin{array}{c}\text { F5 Control } \\
\text { ansiedad interno }\end{array}$ \\
\hline F1 Transferencia & $-.180^{* *}$ & $.128^{* *}$ & $.281^{* *}$ & $.396^{* *}$ & $.317^{* *}$ \\
\hline $\begin{array}{c}\text { F2 Autoconoci- } \\
\text { miento }\end{array}$ & -.034 & $.134^{* *}$ & $.276^{* *}$ & $.296^{* *}$ & $.386^{* *}$ \\
\hline $\begin{array}{c}\text { F3 Construccion } \\
\text { conocimiento }\end{array}$ & $-.111^{*}$ & $.107^{*}$ & $.224^{* *}$ & $.315^{* *}$ & $.371^{* *}$ \\
\hline $\begin{array}{c}\text { F4 Autogestion } \\
\text { aprendizaje }\end{array}$ & $-.149^{* *}$ & $.265^{* *}$ & $.325^{* *}$ & $.608^{* *}$ & $.605^{* *}$ \\
\hline$* * p<.01 * p<.05$ & & & &
\end{tabular}

Para la validez de criterio, se calculó el coeficiente de correlación entre la puntuación de EEAA y ECA de Villardón-Gallego et al. (2013), que mide la competencia para aprender. La correlación es significativa, positiva y moderada $(.487, p<.01)$. Asimismo, tal y como recoge la Tabla 5, se calcularon las correlaciones entre las distintas dimensiones de cada una de las escalas. Como se puede observar, las correlacionas entre los Factores del EEAA y del ECA son significativas y positivas, a excepción de las correlaciones del Factor Evitación del Esfuerzo del EEAA que tiene correlaciones muy bajas y negativas con los Factores del ECA. Los Factores del EEAA que tienen una correlación más alta con las dimensiones de la Competencia para Aprender son la Motivación Intrínseca y el Control de Ansiedad Interno, sobre todo con la Autogestión del Aprendizaje, con quien tienen una correlación positiva moderada.

\section{Discusión y Conclusiones}

Esta investigación ha permitido elaborar y validar un instrumento para medir las estrategias afectivas que utilizan los estudiantes universitarios durante su proceso de aprendizaje. La evaluación de las estrategias afectivas puede contribuir a la regulación de los estados afectivos presentes en los procesos de aprendizaje. Asumiendo que los estudiantes pretenden mejorar sus competencias y mantenerse en un nivel razonable de bienestar (Boekaerts, 2007; Kiener y Weaver, 2011), la evaluación de las estrategias afectivas aporta información útil para ambas pretensiones. Por un lado, facilita información para que los responsables de la formación orienten la enseñanza a mejorar los procesos de aprendizaje y sus resultados, incluyendo la enseñanza intencional de algunas estrategias afectivas o técnicas para que los estudiantes las utilicen estratégicamente (Boekaerts y Corno, 2005; Corno, 2011; Dierdorff, Surface y Brown, 2010; Pekrun, Cusack, Murayama, Elliot y Thomas, 2014). Por otro lado, ayuda a los 
estudiantes a identificar medios para mejorar su propio bienestar durante el aprendizaje y fortalece su motivación para incorporar estrategias útiles para lograrlo.

Para el diseño de la escala, se ha partido de un modelo de cinco dimensiones correlacionadas que se ha mejorado en distintas fases de análisis. En un principio, el instrumento constaba de 44 ítems; se realizó un juicio de expertos para asegurar la validez de contenido, a partir del cual se eliminaron tres ítems por desacuerdo en la asignación a una dimensión. Se realizó un AFC para confirmar la estructura del modelo de 5 factores correlacionados y se fue depurando el instrumento y el modelo para mejorar el ajuste. Así, se eliminaron otros cuatro ítems y la relación entre algunos Factores.

El modelo resultante presenta unos índices de bondad de ajuste muy próximos a .90 , lo que indica que se podría aceptar el modelo teórico de cinco factores, aunque se debería seguir investigando en esta línea hasta conseguir un modelo más ajustado. Las dimensiones que configuran las estrategias afectivas son Evitación del Esfuerzo, Imagen Social, Control Ansiedad Externo, Motivación y Control Ansiedad Interno. A excepción de la Evitación del Esfuerzo, que no correlaciona con el Control de Ansiedad Interno y apenas tiene relación con Motivación, el resto de las dimensiones están correlacionadas de forma positiva; las correlaciones mayores se dan entre Motivación y los dos factores de Control de Ansiedad, Interno y Externo, lo cual puede indicar que estas tres dimensiones configuran un núcleo en cuanto a estrategias afectivas se refiere. Además, se puede deducir que son las más eficaces dada su relación positiva con la Autogestión del Aprendizaje.

El juicio de expertos y la fiabilidad tanto de la escala total como de las dimensiones permiten asegurar la consistencia interna del instrumento. Aunque los coeficientes de fiabilidad son adecuados, habría que mejorar la estructura del factor Control de Ansiedad Externo aumentando el número de ítems. Sería conveniente, asimismo, revisar la pertinencia y formulación de algún ítem de la escala, por ejemplo del ítem 2, que tiene un peso factorial más bajo. Por otro lado, dado que la fiabilidad de la escala es muy alta, sería conveniente, en aras del principio de parsimonia, reducir el número de ítems de Control de Ansiedad Interno y de Imagen Social, de forma que se evitaran posibles solapamientos entre los elementos, y las dimensiones estuvieran más equilibradas en cuanto a extensión. 
Las correlaciones de esta escala de Estrategias Afectivas y sus dimensiones con la escala y dimensiones de la Competencia para Aprender avalan la validez de criterio del instrumento. En este sentido, los datos confirman lo planteado por los estudioso del tema (Zimmerman, 2011), la Motivación Intrínseca y el Control de Ansiedad correlacionan de forma positiva y moderada con la Autogestión del Aprendizaje y la Evitación del Esfuerzo no se relaciona con ninguna dimensión de la Competencia para Aprender. Estos resultados son coherentes con los encontrados por Gargallo, Almerich, Suárez Rodríguez y García-Félix (2012) y por Gil, Bernaras, Elizalde y Arrieta (2009) según los cuales la motivación intrínseca, prioritariamente, y el control de ansiedad interno, en segundo lugar, muestran una fuerte influencia en el aprendizaje estratégico. Asimismo, Bracacevic y Licardo (2010) concluyen el impacto positivo de la autorregulación motivacional en el rendimiento de los estudiantes.

Por otro lado, la ausencia de correlación entre Evitación del Esfuerzo y las dimensiones de Competencia para aprender contrasta con los resultados de Domenech Betoret y Gómez Artiga (2011) según los cuales las estrategias de evitación tienen un papel mediador negativo entre diferentes variables y los resultados de aprendizaje. Ambos resultados sugieren, no obstante, que el uso de estrategias de evitación indica deficiencias en la Competencia para aprender.

En consecuencia, se propone incidir en el uso de estrategias afectivas positivas o adaptativas, es decir, aquellas orientadas a mejorar el aprendizaje y a afianzar la competencia para aprender y sustituir, en la medida de lo posible, las estrategias negativas, es decir, aquellas orientadas a evitar situaciones que exijan esfuerzo o afrontamiento de dificultades para aprender, por alternativas más eficaces para el aprendizaje, coincidiendo con las conclusiones de González, Donolo y Rinaudo (2009).

Por último, cabe mencionar que una de las limitaciones del estudio es la composición de la muestra, con una representación desigual de las diferentes titulaciones de grado y formada por estudiantes de una única universidad. Sería interesante llevar a cabo investigaciones con muestras más amplias y con representación proporcional de distintas instituciones y estudios de grado para validar el instrumento mejorado en base a lo señalado anteriormente. 


\section{Agradecimientos}

Esta investigación forma parte del proyecto financiado por el Ministerio de Ciencia e Innovación del Gobierno de España en convocatoria competitiva Referencia EDU200912883.

\section{Referencias}

Alarcón, G. M., y Edwards, J. M. (2013). Ability and motivation: Assessing individual factors that contribute to university retention. Journal of Educational Psychology, 105(1), 129-137. doi: 10.1037/a0028496

Alvarez, J., Aguilar, J.M. y Lorenzo, J.J. (2012). La Ansiedad ante los Exámenes en Estudiantes Universitarios: relaciones con variables personales y académicas. Electronic Journal of Research in Educational Psychology, 10(1), 333-354.

Brígido, M., Caballero, A., Bermejo, L y Mellado, V. (2009). Las emociones sobre la enseñanza y aprendizaje de las ciencias en estudiantes de Maestro de Primaria. Revista electrónica de motivación y emoción, 11(31). Recuperado de http://reme.uji.es/articulos/numero31/article11/texto.html

Boekaerts, M. (1995). Self-regulated learning - bridging the cap between metacognitive and metamotivation theories. Educational Psychologist, 30(4), 195-200.

Boekaerts, M. (2007). Understanding students' affective processes in the classroom. En P. A. Schutz y R. Pekrun (Eds.), Emotion in education (pp. 37-56). San Diego, CA: Elsevier Academic Press. doi:10.1016/B978-012372545-5/50004-6

Boekaerts, M y Corno, L. (2005). Self-Regulation in the Classroom: A Perspective on Assessment and Intervention. Aplied Psychology: an International Review, 54(2), 199231. doi: 10.1111/j.1464-0597.2005.00205.x

Boza Carreño, A, y Toscano Cruz, M.O. (2012). Motivos, actitudes y estrategias de aprendizaje: aprendizaje motivado en alumnos universitarios. Revista de Currículum y Formación del Profesorado, 16(1), 126-142.

Bracacevic Vukman, K. y Licardo, M. (2010). How Cognitive, Metacognitive, Motivational and Emotional Self-Regulation Influence School Performance in Adolescence and Early Adulthood. Educational Studies, 36(3), 259-268. 
Bueno Garcia, C., Teruel Melero, M.P. y Valero Salas, A. (2005). La Inteligencia Emocional en alumnos de Magisterio: La percepción y comprensión de los sentimientos y las emociones. Revista interuniversitaria de formación del profesorado, 19(3), 169-194.

Corno L. (2011). Studying self-regulation habits. En B. J. Zimmerman, y D. H. Schunk (Eds.), Handbook of self-regulation of learning and performance (pp. 361-375). New York, NY: Routledge.

Dierdorff, E.C., Surface, E.A. y Brown, K.G (2010). Frame-of-Reference Training Effectiveness: Effects of Goal Orientation and Self-Efficacy on Affective, Cognitive, SkillBased, and Transfer Outcomes. Journal of Applied Psychology, 95(6), 1181-1191. doi: $10.1037 / \mathrm{a} 0020856$

Doménech, F. y Gómez-Artiga, A. (2011). Relación entre las necesidades psicológicas del estudiante, los enfoques de aprendizaje, las estrategias de evitación y el rendimiento. Electronic Journal of Research in Educational Psychology, 9(24), 463-496.

Gamboa Araya, R. (2014). Relación entre la dimensión afectiva y el aprendizaje de las matemáticas. Revista Electrónica Educare, 18(2), 117-139. doi: 10.15359/ree.18-2.6

García-Ros, R., Pérez-González, F., Pérez-Blasco, J y Natividad, L.A. (2012). Evaluación del estrés académico en estudiantes de nueva incorporación a la universidad. Revista Latinoamericana de Psicología, 44(2), 143-154. doi: 10.14349/rlp.v44i2.1038

Gargallo, Bernardo, Almerich, Gonzalo, Suárez-Rodríguez, Jesús M. y García-Félix, Eloina (2012). Estrategias de aprendizaje en estudiantes universitarios excelentes y medios. Su evolución a lo largo del primer año de carrera. RELIEVE, 18(2), art. 1. doi: 10.7203/relieve.18.2.2000

Garritz, A. (2010). Personal Reflection: Pedagogical Content Knowledge and the Affective domain of Scholarship of Teaching and Learning. International Journal for the Scholarship of Teaching and Learning, 4(2), Article 26. Recuperado de http://digitalcommons.georgiasouthern.edu/ij-sotl/

Gil. N., Blanco, L.J. y Guerrero, E. (2005). El dominio afectivo en el aprendizaje de las matemáticas. Una revisión de sus descriptores básicos. Revista Iberoamericana de educación matemática, 2, 15-32. Recuperado de http://www.fisem.org/www/union/ (2006). El papel de la afectividad en la resolución de problemas matemáticos. Revista de Educación, 340, 551-569.

Gil, P., Bernaras, E., Elizalde, L.M. y Arrieta, M. (2009). Estrategias de aprendizaje y patrones de motivación del alumnado de cuatro titulaciones del Campus de Gipuzkoa. 
Infancia y Aprendizaje: Journal for the Study of Education and Development, 32(3), 329-341. doi: 10.1174/021037009788964132

González, A., Donolo, D. y Rinaudo, C. (2009). Emociones académicas en universitarios: su relación con las metas de logro. Ansiedad y estrés, 15(2-3), 263-277.

Hervás, G. y Vázquez, C. (2006). La regulación afectiva: Modelos, investigación e implicaciones para la salud mental y física. Revista de Psicología General y Aplicada, 59(1-2), 9-36.

Hugo, D., Sanmartí, N. y Adúriz-Bravo, A., (2013). Estilos de trabajo emocional del futuro profesorado de ciencias durante el prácticum. Enseñanza de las Ciencias, 31(1), 151168.

Kiener, M. y Weaver, C. (2011). Examining How Cognitive And Affective Learning Strategies Change As Students Complete Coursework. Transformative Dialogues: Teaching \& Learning Journal, 5(1), 1-12. Recuperado de http://www.kpu.ca/td/past-issues/5-1

Koballa, T.R. y Glynn, S.M. (2007). Attitudinal and Motivational constructs in science learning. En S. K. Abell, y N. G. Lederman, (Eds.). Handbook of Research on Science Education, (pp. 75-102). Mahwah, NJ, USA: Erlbaum.

Marins, P. R., y Guijarro, J. R. (2010). Afectividad y competencia existencial en estudiantes de español como lengua extranjera en brasil. RLA, Revista de lingüística teórica y aplicada, 48(1), 51-74. doi: 10.4067/S0718-48832010000100004

Marcos-Llinàs, M. (2007). Variables afectivas en la clase de lenguas extranjeras. Interlingüística, 17, 676-678.

Núñez, J. C., Valle, A., Cabanach, R. G., González-Pienda, Julio A., Rodríguez, S., Rosario, P. (2009). Perfiles motivacionales en estudiantes universitarios. Revista de Psicología y Educación, 1(4), 15-30.

Nuñez, J.C. y González-Pienda, J.A. (1994). Determinantes del rendimiento académico. Variables cognitivo-motivacionales, atribucionales, usos de estrategias y autoconcepto. Oviedo: Universidad de Oviedo.

Páez, D. y Carbonero, A.J. (1993). Afectividad, cognición y conducta social. Psicothema, 5(suplemento), 133-150.

Palacios, A., Hidalgo, S., Maroto, A. y Ortega, T. (2013). Causas y consecuencias de la ansiedad matemática mediante un modelo de ecuaciones estructurales, Enseñanza de las Ciencias, 31(2), 93-111. 
Panadero, E. y Alonso-Tapia, J. (2014). ¿Cómo autorregulan nuestros alumnos? Revisión del modelo cíclico de Zimmerman sobre la autorregulación del aprendizaje. Anales de Psicología, 30(2), 450-462. doi: 10.6018/analesps.30.2.167221

Pekrun, R., Maier, M. A. and Elliot, A. J. (2009). Achievement goals and achievement emotions: Testing a model of their joint relations with academic performance. Journal of Educational Psychology, 101(1), 115-135. doi: 10.1037/a0013383

Pekrun, R, Cusack, A., Murayama, K., Elliot, A.J. y Thomas, K. (2014). The power of anticipated feedback: Effects on students' achievement goals and achievement emotions. Learning and Instruction 29, 115-124. doi: 10.1016/j.learninstruc.2013.09.002

Putwain, D. (2007). Researching academic stress and anxiety in students: some methodological considerations. British Educational Research Journal, 33(2), 207-219. doi: $10.1080 / 01411920701208258$

Schutz, P.A. and Zembylas, A. (Eds.)(2009). Advances in Teacher Emotion Research: The Impact on Teachers' Lives. EEUU: Springer. doi: 10.1007 / 978-1-4419-0564-2

Suárez, J. M. y Fernández, A. P. (2005). Escalas de evaluación de las estrategias motivacionales de los estudiantes. Anales de Psicología, 21(1), 116-128.

Suárez, J. M., y Fernández, A. P. (2011). Evaluación de las estrategias de autorregulación afectivo-motivacional de los estudiantes: Las EEMA-VS. Anales de Psicología, 27(2), 369-380.

Valle, A., Núñez, J.C., González-Cabanach, R., Rodríguez-Rodríguez, S., González-Pienda García, J.A. y Rosário, P. (2007). Metas académicas y estrategias motivacionales de autoprotección. Electronic Journal of Research in Educational Psychology, 5(3), 617632.

Valle, A., Rodríguez-Martínez, S. González-Cabanach, R., Núñez, J.C., González-Pienda García, J.A. y Rosário, P. (2009). Metas académicas Perspectiva histórica y conceptual e implicaciones académicas. ElectronicJournal of Research in Educational Psychology 7(3), 1073-1106.

Vázquez, S.M. (2009). Motivación y voluntad. Revista de Psicología, 27(2), 185-212.

Villardón-Gallego, L., Yániz, C., Achurra, C., Iraurgui, J. y Aguilar, M.C. (2013). Learning Competence in University: Development and Structural Validation of a Scale to Measure. Revista de Psicodidáctica, 18(2), 357-374. doi: 10.1387/RevPsicodidact.6470

Walshaw, M y Brown, T (2012). Affective productions of mathematical experience. Educational Studies in Mathematics, 80(1-2), 185-199. 
Zembylas, M (2007). Emotional ecology: The intersection of emotional knowledge and pedagogical content knowledge in teaching. Teaching and Teacher Education, 23(4), 355367. doi: 10.1016/j.tate.2006.12.002

Zimmerman, B.J. (2011). Motivational sources and outcomes of self-regulated learning and performance. En B. J. Zimmerman, \& D. H. Schunk (Eds.), Handbook of selfregulation of learning and performance (pp. 49-63). New York, NY: Routledge. 